\title{
MUJERES AFECTADAS POR EL NEMAGÓN: LA ORGANIZACIÓN PARA REGISTRAR EL SUFRIMIENTO AMBIENTAL ${ }^{1}$
}

\section{WOMEN AFFECTED BY NEMAGÓN: ORGANIZATION TO REGISTER ENVIRONMENTAL SUFFERING}

\author{
Sindy Mora Solano*
}

\begin{abstract}
RESUMEN
En este artículo se analiza el proceso de organización de mujeres trabajadoras bananeras afectadas por el agroquímico conocido como nemagón (DCBP). En los años 90, estas mujeres se organizaron con la finalidad de registrar los padecimientos en sus cuerpos producto de la exposición a los agroquímicos. Para ello, se consulta la literatura en el tema y se analizan fuentes del año 1996 del archivo de la organización Servicio Paz y Justicia (SERPAJ).
\end{abstract}

PALABRAS CLAVE: MOVIMIENTOS SOCIALES * TRABAJADORAS * PLANTACIÓN * AGROQUÍMICOS * SALUD

\section{ABSTRACT}

This article analyzes the process of organizing female banana workers affected by the pesticide known as Nemagón (DCBP). In the 1990s, these women organized themselves with the purpose of recording the suffering in their bodies as consequence of the exposure to pesticides. For this, are analyzed the literature on the subject and sources of 1996 from the archive of the organization Paz y Justicia (SERPAJ).

KEYWORDS: SOCIAL MOVEMENT * WOMEN WORKERS * PLANTATION * PESTICIDES * HEALTH

Instituto de Investigaciones Sociales de la Universidad de Costa Rica.

sindymora@gmail.com

1 Agradezco la colaboración brindada por Ana Lucía Castro y Gustavo Cabrera del Servicio Paz y Justicia-Costa Rica (SERPAJ) para la elaboración de este artículo; así como, el trabajo realizado por Valeria Vargas y Shirly Chocrón. De la misma manera, agradezco los comentarios del Dr. Mauricio Menjívar Ochoa a la ponencia presentada en el Instituto de Investigaciones Sociales (IIS) de la Universidad de Costa Rica. 


\section{INTRODUCCIÓN}

Este artículo forma parte de los resultados del proyecto de investigación "Envenenando la salud, potenciando la participación política: las mujeres afectadas por el nemagón en la Región Caribe de Costa Rica, entre 1990-2010", inscrito en el Instituto de Investigaciones Sociales (IIS) de la Universidad de Costa Rica.

A partir del trabajo de investigación previamente realizado, en el que se ha indagado en torno a las principales características del movimiento de la población de trabajadoras bananeras (Mora, 2013, 2016a y 2016b), en este artículo se estudia uno de los esfuerzos organizativos de mujeres afectadas por el DiBromoCloroPropano (DBCP), más conocido con el nombre comercial de nemagón. Esta iniciativa surgió en los años 90 , en una de las coyunturas más álgidas de este movimiento, con el apoyo de la organización no gubernamental Servicio Paz y Justicia Costa Rica (serpas). A partir de la articulación con serpas, las mujeres juntaron esfuerzos para darle cabida a la reflexión en torno a su dolor. El dolor es analizado a partir de las discusiones referidas al sufrimiento ambiental, concepto elaborado por el sociólogo Javier Auyero y la antropóloga Débora Swistun (2008). El sufrimiento ambiental:

es (junto con una indagación sobre los "datos duros" de la contaminación) un examen de las experiencias y los sentidos atribuidos a este sufrimiento. Una etnografía del sufrimiento ambiental es un análisis de las voces de quienes padecen, pero es también un estudio de las narrativas que circulan alrededor de las vidas de quienes lo padecen, esto es, de todos los intentos de darle sentido a ese sufrimiento, de todas las apropiaciones y reconocimientos que son, como lo implica el análisis, actos profundamente políticos (Auyero y Swistun, 2008, p.218).

Como se observa, el sufrimiento ambiental es un concepto que invita a la escucha $y$ al reconocimiento de aquellas voces que experimentan un dolor, sin que necesariamente este se encuentre reconocido en los planos jurídicos, médicos, organizativos o sociales vinculados a la construcción de las memorias relacionadas a este (Kleinman y Kleinman, 1997). En este sentido, Veena Das (2008) ha señalado la necesidad de investigar cómo se recuperan y se posicionan en la memoria, las experiencias traumáticas de dolor, tanto individuales, locales $y$ nacionales. Haciendo referencia a las experiencias traumáticas vinculadas a la violencia sexual vivida por las mujeres, esta autora señala:

Nunca estamos seguros de si la distancia de estas imágenes es una ilusión óptica, pues siempre existe la tentación, como sucede en la narrativa de familia y en las narrativas nacionalistas, de expulsar estas imágenes de la ribera de la experiencia cotidiana hacia un horizonte distante e invisible. Sin embargo, debemos preguntar qué efectos tuvo esta brutalización para las experiencias de la identidad personal, la comunidad y la nación (2008, p. 345).

Tomando en cuenta estos referentes, este artículo se compone de tres apartados. En el primero, se describe la metodología utilizada $y$ se caracterizan las fuentes por analizar. En el segundo, se realiza una contextualización de la situación de las mujeres y su exposición a los agroquímicos. En el último, se analiza la experiencia de las mujeres afectadas por el nemagón, en su esfuerzo por registrar su sufrimiento ambiental.

\section{METODOLOGÍA}

El 9 de junio de 1996, un grupo de mujeres que se autodenominaron afectadas por el nemagón participaron en una reunión en la que registraron sus padecimientos, producto de la exposición a este agroquímico. En esta reunión, convocada por SERPAJ, se produjeron una serie de documentos en los que se exponen las experiencias de estas mujeres. Los registros de la afectación en la salud de las participantes 
de esta actividad han sido conservados en el archivo del SERPAJ ${ }^{2}$.

Los documentos disponibles son 53 textos, en papeles de colores de una textura muy suave, algunos casi transparentes y escritos a mano, en donde se indica el nombre de la trabajadora bananera, el número de cédula, en algunas ocasiones la fecha de nacimiento y por ende, la edad (la que, por lo general, aparece ilustrada mediante una resta), la dirección de la casa de habitación, el número de hijos vivos, el número de hijos muertos, el número de abortos y los padecimientos. En algunos casos, ha sido posible detectar que otra persona escribió el texto, por la caligrafía y el tiempo verbal utilizado, lo que lleva a plantear la hipótesis sobre el analfabetismo o baja escolaridad de las mujeres que participaron en la actividad. Sin embargo, es importante señalar que estos casos son los menores, dado que la mayor parte de los documentos se encuentran escritos en primera persona singular. Muchos de los textos fueron escritos con lápiz, lo que ha dificultado que se preserven los rasgos de las escrituras en óptimas condiciones; algunos se encuentran escritos con lapicero. Todos los documentos están firmados por las mujeres participantes.

Con el fin de reproducir y escuchar lo que estas mujeres plantearon sobre sus cuerpos y su sufrimiento, se ha procedido a transcribir cada uno de estos materiales, omitiendo el nombre de la persona que escribe, el nombre de las hijas y los hijos, el número de cédula y la dirección de sus casas. De estos 53 documentos, 51 han sido transcritos, dado que, por la caligrafía, no fue posible transcribir dos de estos.

La existencia de este material viene a confirmar la tesis de que las mujeres fueron organizadoras y partícipes de una serie de iniciativas en su proceso de afectación por los agroquímicos, a pesar de que estas actividades no necesariamente, se hicieron de conocimiento

$2 \quad$ A partir de la indagación realizada, se puede señalar que estos documentos no han sido analizados previamente. A pesar de que existe un informe producto de una pasantía realizada por un estudiante de la Universidad Nacional (UNA), en donde se estudia la labor de SERPAJ (Cárdenas, s.f), dicho informe no hace referencia a este material. público o fueron opacadas por un movimiento que, tradicionalmente, se consideró como un movimiento de trabajadores (Mora, 2016b).

Valga señalar que una de las hipótesis en torno al momento en el que surge el movimiento de afectados por el nemagón, a principios de los años 90, se relaciona con la dificultad que pudieron tener los protagonistas de este proceso, es decir, los hombres, para hablar de lo que estaba sucediendo en sus cuerpos (Mora, 2016b). Lo anterior, a pesar de que los primeros casos de afectación se dieron a finales de los años 70 (Giralt, 1996). La existencia de estos documentos escritos permite posicionar el lugar de la palabra en la discusión colectiva sobre las distintas expresiones de un sufrimiento, socialmente compartido, aunque no necesariamente reconocido, que en este caso es el de las mujeres.

Para este trabajo se realizaron dos entrevistas con personas involucradas a esta iniciativa. Una realizada con el coordinador de SERPAJ $y$ otra con una extrabajadora bananera, quien ahora tiene 75 años de edad y que estuvo presente en la reunión del 9 de junio de 1996. La dificultad para encontrar mujeres que participaron en esta iniciativa se relaciona no solo con el distanciamiento que ellas han vivido con esta forma organizativa, sino con la edad que actualmente tienen (algunas mujeres de las que se tiene registro fallecieron).

\section{LO QUE SE SABE SOBRE LA EXPOSICIÓN DE LAS MUJERES A LOS AGROQUÍMICOS}

La experiencia de afectación por el uso de los agroquímicos no es exclusiva de las mujeres expuestas al рвср. En la actualidad existe un amplio conocimiento en torno a los efectos de los agroquímicos, tanto en el ambiente como en los distintos grupos sociales expuestos a estas sustancias, lo cual no niega la necesidad de una mayor investigación.

En términos generales, distintos estudios han demostrado que cuando las mujeres se encuentran incorporadas a las dinámicas laborales, ya sean formales o informales, se observa una limitada regulación de la distribución, el transporte, el almacenamiento y el uso de agroquímicos; una falta de entrenamiento 
o capacitación en cuanto a su uso, una limitada capacidad de instrucción e incluso, de lectura, lo que conlleva a una reducida posibilidad de dar seguimiento a las instrucciones en las etiquetas de las sustancias que se van a utilizar; un uso inadecuado del equipo de protección o la inexistencia de este, carencia de lugares apropiados para el aseo personal después de realizar las labores correspondientes o el lavado de la ropa, aunado a la ausencia de centros médicos cercanos que permitan atender las necesidades de la población (Dinham, 2003).

Los estudios de caso específicos muestran la profundización de los rasgos señalados. En el estudio realizado por Nivia $(2000)^{3}$, en el municipio de Palmira, en Colombia, se evidencia el uso excesivo de agroquímicos en las plantaciones, al que se unen las aplicaciones aéreas de estas sustancias. Aunque los hombres se encuentran mayoritariamente expuestos a los agroquímicos, los menores de edad y las mujeres también lo están, a pesar de no encontrarse vinculados directamente a las labores de riego, tal y como lo muestran los estudios médicos. Si bien, se necesita mayor indagación, a partir de la muestra del estudio, la autora señala que la exposición a estas sustancias por parte de las mujeres en edad reproductiva que desempeñan estas labores, puede ser un factor asociado a las altas tasas de aborto, malformaciones congénitas $y$ niños nacidos muertos.

En el caso de la floricultura en Ecuador, la expansión de este monocultivo en los años 90 transformó las relaciones sociales, incorporando mano de obra entre los 15 y los 35 años, fundamentalmente mujeres, con empleos mal remunerados, con largas jornadas laborales $y$ con una alta exposición a los agroquímicos (Korovkin, 2004) ${ }^{4}$. A pesar de disponer de equipo que protege de la exposición a estas sustancias, este no es de la mejor calidad, ya que se rompe

3 En esta investigación, se realizaron observaciones de campo, encuestas y entrevistas con personas de la comunidad; adicionalmente, se realizaron estudios médicos con la población de estudio.

4 Para esta investigación, la autora realizó trabajo de campo con trabajadores $y$ trabajadoras floricultoras, trabajadoras migrantes $y$ amas de casa de una zona florícola de la Sierra ecuatoriana. al utilizarse e incluso, en algunas ocasiones las trabajadoras prefieren no usarlo, debido a que se convierte en un obstáculo para realizar rápidamente las labores asignadas. La investigación muestra cómo la preocupación por la exposición a los agroquímicos es mayor entre la población de la zona florícola de la Sierra, que la que tienen las personas que trabajan con estas sustancias.

En el estudio de Reeves, Murphy y Calvo $(2003)^{5}$, realizado con trabajadoras vinculadas a la Organización en California de Líderes Campesinas y la United Farmworkers of America (ufw), en California, Estados Unidos, se muestran las dificultades a las que se enfrentan las mujeres que aplican agroquímicos. Al ser la mayor parte de estas trabajadoras migrantes mexicanas (91\%), con un limitado manejo del inglés (83\% no maneja el idioma), uno de los principales problemas es el gran nivel de subregistro de las intoxicaciones sufridas en el proceso de aplicación. Entre las razones por las que las mujeres no reportaron su afectación (23\%), se destaca el miedo a perder el trabajo, los altos costos de los servicios médicos, las dificultades para transportarse a los centros de salud y el desconocimiento de los síntomas de la intoxicación, que pueden ser confundidos con otras enfermedades, como intoxicaciones por la ingesta de alimentos. Entre los principales síntomas reportados están sarpullidos, alergias, dolores de cabeza, mareos $y$ vómitos. El estudio muestra que muchas de las intoxicaciones quedan sin atender o son atendidas mediante remedios caseros.

El trabajo publicado por la Asociación de Servicios de Promoción Laboral (aseprola) $(2003)^{6}$ recoge las experiencias de las mujeres bananeras centroamericanas, quienes relatan las condiciones laborales y organizativas en las

$5 \quad$ En este estudio, las autoras aplicaron un instrumento de encuesta diseñado conjuntamente por Pesticide Action Network North America (PANNA), la Organización en California de Líderes Campesinas y la United Farmworkers of America (UFW). El instrumento fue contestado por 47 trabajadoras, en su mayoría, migrantes mexicanas.

6 ASEPROLA recopiló historias de vida de mujeres trabajadoras bananeras de Centroamérica. 
que se encuentran. Si bien no es un trabajo que analice de manera específica la exposición de las mujeres a los agroquímicos, en este se pueden retomar los padecimientos de las mujeres debido a su exposición a estas sustancias, entre las que se encuentran: "afecciones respiratorias, enfermedades de la piel, hongos en pies y manos, pérdida de la voz, alteración en venas, dolores de vientre $y$ dolores en los huesos" (2003, p. 104).

En el texto de Frank (2006) se exponen las condiciones laborales y las formas organizativas de las mujeres bananeras en América Latina, brindando especial atención a las mujeres de Centroamérica. En el estudio se mencionan procesos de afectación en la salud, entre los que se encuentran:

(...) heridas por movimientos repetitivos, $y$ enfermedades en la piel causadas por la exposición a los químicos y el agua [...] lesiones en la espalda, artritis prematura y caídas en los centros de trabajo. Las mujeres reportaron, además, altas tasas de abortos y tipos de cáncer poco comunes entre las trabajadoras, la mayoría sin contratos colectivos y con poco o ningún acceso a atención médica (p. 47).

En el caso de las comunidades indígenas y quilombolas brasileñas del Estado de Espírito Santo afectadas por la expansión del eucalipto a partir de los años 60 (Barcellos y Batista, $2007)^{7}$, las autoras muestran cómo el despojo de sus tierras trajo consigo cambios en las formas productivas, lo que extinguió actividades como la siembra, la caza y la pesca, aumentando la exposición de la población a los agroquímicos y por ende, los padecimientos como las alergias y afecciones en la piel.

El estudio realizado por Camarena y otras $(2013)^{8}$ muestra cómo las mujeres indígenas que

$7 \quad$ Las autoras realizaron entrevistas a mujeres indígenas y quilombolas del Estado de Espírito Santo, Brasil.

8 Las autoras realizaron observaciones participantes, entrevistas grupales y talleres con mujeres indígenas que trabajan en Ensenada, Baja California. laboran en Ensenada, Baja California, México, trabajan jornadas extenuantes de hasta 14 horas $y$ deben llevar sus propios implementos de seguridad, como guantes o pañoletas para protegerse de los "líquidos", nombre con el que llaman a los agroquímicos. Adicionalmente, deben comer en los lugares de trabajo, lo que expone sus alimentos a la contaminación. Entre los efectos en la salud que reporta la investigación se encuentran irritaciones, manchas y ronchas en la piel, así como, dolores de cabeza, náuseas y vómitos.

Por su parte, González (2014) analiza el proceso de producción de flores en la Sabana de Colombia. La autora muestra cómo las trabajadoras han sido expuestas a 127 tipos de pesticidas, muchos de los cuales se encuentran prohibidos en países como Estados Unidos, principal destinatario de las flores.

Finalmente, el estudio realizado por Van Wendel $y$ otros $(2014)^{9}$ analiza para el caso de Costa Rica, las altas concentraciones de etilentiourea (Ети) que tienen en su orina las mujeres embarazadas que viven cerca de las plantaciones de banano en Matina, en la provincia de Limón. El Eтu es utilizado para combatir la sigatoga negra y se riega vía aérea, lo que explica las altas concentraciones de esta sustancia, incluso, en la población que no se encuentra vinculada de manera remunerada a las bananeras.

Valga subrayar que un aspecto aportado por varias de las investigaciones reseñadas es la necesidad de realizar estudios médicos que permita conocer, a profundidad, la situación de salud en la que se encuentran las comunidades y las mujeres (Nivia, 2000; Korovkin, 2003). Como señalan distintos estudios, las mujeres se encuentran en una situación de mayor vulnerabilidad, dado que cuentan con limitadas condiciones de organización o afiliación a las organizaciones existentes, como los sindicatos (Aseprola, 2003; Korovkin, 2003; Frank, 2006; Valdés, 2015).

$9 \quad$ Para esta investigación se realizaron análisis de muestra de orina de 445 mujeres. Si bien, un grupo de estas mujeres se desempeñaba como trabajadoras bananeras, la mayor parte de estas vivía cerca de los bananales, pero no se encontraba incorporada remuneradamente a las bananeras. 
Aunque algunas mujeres han guardado silencio, otras se han organizado para luchar contra la exposición a los agroquímicos. En Brasil, por ejemplo, destacan las experiencias protagonizadas por mujeres vinculadas a la lucha contra los sembradíos de eucalipto, en alianza con organizaciones feministas (Siliprandi, 2011) ${ }^{10}$; así como es significativa la experiencia de agrupaciones de mujeres en Colombia, que se han dedicado a la producción para el autoconsumo y la venta local (Zuluaga y Arango, 2013) ${ }^{11}$.

Estas iniciativas, a menudo concebidas "pequeñas" en el marco de las transformaciones necesarias para enfrentar problemáticas sociales como el uso y exposición a los agroquímicos, permiten plantear la pregunta en torno a los mecanismos con los que, en ocasiones, se evalúa la participación política de las mujeres:

a pesar de la relevancia de estas asociaciones, la sociedad en general no las concibe como un actor político protagónico, debido a que siempre se las asocia con su papel doméstico y de auxiliadoras en distintos tipos de crisis, es decir, cuando reproducen los roles "propios" de su género: madres, esposas, enfermeras, trabajadoras sociales, etc. (Zuluaga y Arango, 2013, p. 171; cursivas en el original).

En este sentido, a pesar de que el esfuerzo organizativo que se presenta seguidamente es una iniciativa pequeña, leída en el contexto del surgimiento del movimiento de trabajadores bananeros afectados por el nemagón adquiere un carácter especial, ya que permite escuchar $y$ hacer visible el posicionamiento

10 Para esta investigación, la autora registra las principales marchas y reivindicaciones de las mujeres de Espírito Santo, Brasil, conocidas como las Marchas das Margaridas, en honor a Margarida Maria Alves, lideresa sindical rural asesinada en 1983.

11 Las autoras realizaron entrevistas y talleres con mujeres de la Asociación de Mujeres Organizadas de Yolombó (АмOY). político de las mujeres en la búsqueda de su reconocimiento (Auyero, 2004).

ESFUERZOS ORGANIZATIVOS PARA REGISTRAR EL SUFRIMIENTO AMBIENTAL

\section{EL SURGIMIENTO DE LA INICIATIVA ORGANIZATIVA}

La investigación realizada previamente ha mostrado cómo el movimiento de la población trabajadora bananera afectada por el nemagón estableció una jerarquía de demandas, en la que la indemnización económica por la esterilidad masculina articuló las distintas acciones realizadas (Mora, 2016b). Mediante este proceso se dejaron de lado otras reivindicaciones, como los servicios de salud especiales para los afectados directos e indirectos, fueran estas esposas, hijos e hijas de esta población; estudios para conocer con certeza los eventuales problemas de salud que podía desarrollar la población afectada o pensiones especiales para este tipo de trabajadores. De igual forma, este proceso posibilitó el surgimiento y desarrollo de una serie de actores, como bufetes de abogados y "gavilanes", que se dieron a la tarea de cobrar dinero para "llevar los casos"12 en contra de las empresas extranjeras y ante el Instituto Nacional de Seguros (INs), en muchas ocasiones lucrando con el dolor de la población afectada por los agroquímicos (Mora, 2016b).

Es por ello que en los años 90, cuando se empieza a hablar de las posibilidades de indemnizar a esta población, antes de que se aprobara la legislación con la que se indemnizaría a través del ins en el año 2001, las mujeres que se encontraban en ese proceso se vieron excluidas de la posibilidad de acceder a una indemnización por parte de empresas transnacionales, ya que se decía que únicamente los hombres habían sido afectados por dicha sustancia (Comunicación personal, coordinador

12 "Llevar el caso" es la expresión utilizada para hacer referencia al proceso mediante el cual los abogados o los llamados "gavilanes" se encargan del proceso jurídico de las personas afectadas por el nemagón, con la finalidad de conseguir una indemnización. 
SERPAJ, 25/05/2016). Es en este contexto que SERPaJ se propuso organizar una serie de sesiones para hablar con las mujeres y empezar a registrar los padecimientos de quienes estaban afectadas por el DBCP, estrategia que brindó un espacio para que estas pudieran conversar de lo que les estaba sucediendo.

Si bien no existen registros de otras iniciativas similares en el archivo de serpas, el coordinador de esta organización señalaba cómo dichas actividades se realizaron durante año y medio, con la finalidad de levantar un registro de las mujeres que se encontraban afectadas por el agroquímico, como lo muestra la siguiente transcripción:

- (...) y decidimos ir los sábados y los domingos durante ese año, año y medio incluso, a los lugares, a las comunidades, verdad, en Cariari, allá en Los Patios de San Cristóbal, en Guácimo, Siquirres, ir a hacer asambleas a las comunidades porque mucha gente no estaba informada y segundo porque era importante llegar donde estaba la gente afectada porque es un daño, una enfermedad, una enfermedad que queda $y$ que la persona por vergüenza, porque no tiene ánimo, porque no tiene fondos, gente muy pobre que estaba muy enferma, que no podía salir, era más fácil llegarle, adonde estaban desde la perspectiva de los derechos humanos. Entonces fuimos ahí y encontramos muchas mujeres que nos decían que tenían hemorragias vaginales permanentes, abortos consecutivos, los niños nacían con deformaciones congénitas, los lacrimales permanentemente sueltos, o sea, siempre llorando, exemas en la piel, sus hijos también cuando crecían, crecían con escamas en la piel, con sarpullidos, surpuraciones en el oído o en el ojo, qué se yo, no sé si usted tendrá un atestado de eso. Igual en el serpaj hay entrevistas a mujeres (Comunciación personal, coordinador SERPAJ, 25/05/2016).
En el marco de esta iniciativa $y$ en sesiones posteriores, se hicieron talleres de autoestima $y$ se realizaron sesiones en las que las mujeres recibieron servicios de salud de acupuntura. Si bien las mujeres se siguieron reuniendo, el acompañamiento y el encuentro colectivo fue uno de los principales resultados de esta iniciativa. Los documentos en los que se basa este artículo son una expresión de este proceso.

\section{¿QUÉ DICEN LAS MUJERES AFECTADAS POR EL NEMAGÓN?}

Los documentos que se encuentran en el archivo de seRpas dan cuenta de un intento de sistematización de las expresiones corporales del sufrimiento ambiental. Como se puede ver en la imagen 1, en la reunión realizada el 9 de junio de 1996, las mujeres buscaron darle lugar al dolor, identificando una serie de padecimientos que ellas vincularon a su exposición al agroquímico. En esta imagen se puede observar un levantamiento hecho a mano de la información proveniente de los cuerpos de las mujeres que participaron en la actividad. Con este mecanismo, se contabilizaron los números totales de mujeres que desarrollaron abortos, problemas vaginales, problemas en los ojos, los huesos, alergias, hongos, migrañas y que tuvieron hijos e hijas con problemas de salud.

En la entrevista realizada con una extrabajadora bananera que fue parte de esta iniciativa, señaló que las convocatorias para participar en estas reuniones eran abiertas y se realizaron a través de Radio Guápiles. Los encuentros se llevaron a cabo en La Emilia, tradicional salón que se ubica en el mismo distrito (Comunicación personal, extrabajadora bananera, 20/07/2016). Si bien, esta organización tenía como finalidad emprender los procesos judiciales en contra de las empresas transnacionales, estos no pudieron llevarse a cabo, dado que las empresas bananeras dejaron claro que las indemnizaciones se iban a realizar únicamente con los hombres que hubiesen estado expuestos al nemagón y que demostraran su esterilidad. 
IMAGEN 1

RECUENTO MANUAL DE PADECIMIENTOS DE 53 MUJERES AFECTADAS POR EL NEMAGÓN

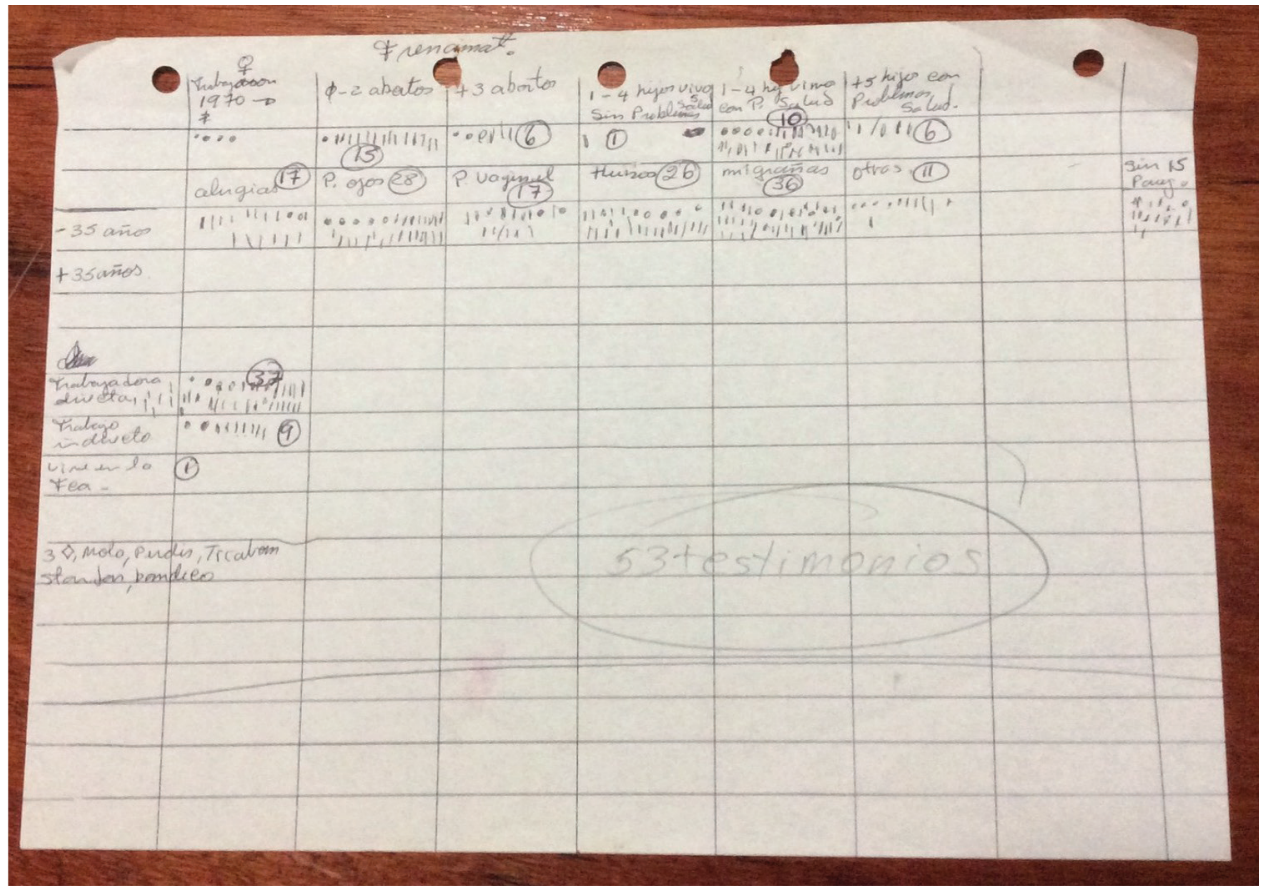

Fuente: $\quad$ SERPAJ, 1996.

A partir del material analizado, se plantea una discusión sobre los principales temas que se presentan en los documentos provenientes del archivo de sERPaJ, escritos por las trabajadoras bananeras. Entre estos se tratarán los siguientes: 1. Mujeres afectadas directa e indirectamente. 2. Los abortos como principal consecuencia de la exposición al nemagón y 3. El nemagón como explicación de todos los padecimientos. Valga señalar que los relatos han sido numerados de acuerdo al orden en que aparecen en el expediente consultado de SERPAJ, numeración que se conserva en este artículo.

\section{MUJERES AFECTADAS DIRECTA E INDIRECTAMENTE}

En los documentos revisados es posible identificar a las mujeres que se vieron afectadas de manera directa por el agroquímico de aquellas que fueron afectadas indirectamente, dado que tuvieron contratos laborales limitados en el tiempo y posteriormente, se dedicaron a las labores del hogar en sus casas, manteniéndose en contacto con esta sustancia.

Un ejemplo del primer caso aparece en el relato 2 , en donde la mujer narra las consecuencias de su exposición a los agroquímicos después de trabajar en la empresa Ticaban. Como se puede observar, la mujer señala que tuvo 8 hijos y 3 abortos, así como indica los problemas que desarrollaron tanto ella como su compañero para vivir su sexualidad.

Relato 2. Mujer nacida el 28 de marzo de 1943

Hijos 8 vivos 3 ovortos.

Yo la afectada pongo en comunicación [...no se entiende la letra...] de que padezco de dolores de cabeza dolor de hueso los dedos perjudicados que nunca 
tuve cura salí con la vista corta y tengo ocho operaciones a consecuencia del trayecto del trabajo $y$ diario me mantengo como un desconsuelo en el cuerpo. Y también creo como el sexo tengo como 4 años que tengo el compañero pero los estamos iguales porque el también está en lo mismo y por eso no puede estar en la esterilización porque no puede hacer el examen.

Yo trabaje en Tica Ban quince años fui liquidadada en el año 1985 (sERPAJ, 1996).

En este relato, la mujer hace referencia a la imposibilidad de su compañero de tener una erección, con el fin de obtener una muestra de semen para realizarse el examen que solicita el INs $y$ que permite determinar si es afectado por el nemagón. La esterilidad es señalada como una de las consecuencias directas de la exposición al agroquímico, como también se puede observar en el relato 39, en el que la mujer identifica como consecuencia directa la esterilidad suya y la de su esposo. Adicionalmente, ella señala otros problemas de salud.

Relato 39. Documento sin fecha de nacimiento

Empezé a trabajar en el 69 en Valle de la Estrella con Standard Fruit.

70 trabajana en Bandeco - Finca San Peter hasta el 72.

todo tipo de trabajo (selectar, pacas, sellar...) Nemagon y otros (uno que olía muy feo)

Despues del 72 me operarón y me dijeron despues que estaba estéril.

Mi esposo también está estéril.

dolor de cabeza, cintura, espalda, huesos, presión alta

Pbma asma (serpaJ, 1996).

Ahora bien, una muestra del segundo caso, es decir, de personas afectadas indirectas, se presenta en el relato 3 . La entrevistada señala que ella estuvo vinculada a las bananeras desde el 68 al 69. Posteriormente, se dedicó a las labores domésticas, entre las que se encontraba lavar la ropa de su esposo. Si bien, ella trabajó un año en la bananera, al salir de trabajar remuneradamente, continuó expuesta a los agroquímicos por las labores domésticas.

Relato 3. Mujer nacida el 23 de diciembre de 1953

Mi enfermedad son: dolor de cuerpo, cabeza y padesco de la garganta, la presión, nervios, mareos, la vista tuve un aborto

Yo lavava la ropa a mi esposo cuando iva a regar los quimicos del banano

Yo tambien trabaje 68 y Sali 69

Tengo 3 hijos [...nombres de los hijos...] Mi hijo mayor tubo problemas en el aprendizaje nacio ante de tiempo le dio patites a mi y a el. Él tiene un poquito de retardo (sERPAJ, 1996).

A pesar de la distinción señalada, es central indicar que en el caso de los documentos analizados, la mayor parte de las mujeres son afectadas directas, justamente, por su vinculación como trabajadoras de las bananeras.

\section{LOS ABORTOS COMO PRINCIPAL CONSECUENCIA DE LA EXPOSICIÓN AL NEMAGÓN}

Una de las secuelas más comunes nombradas en los documentos analizados son los abortos. De 53 relatos estudiados, 18 mujeres señalaron haber tenido abortos; algunas de estas mujeres tuvieron un único aborto, mientras que otras tuvieron un número mayor de pérdidas. Por ejemplo, en el relato 5 , la mujer que cuenta su historia señala haber vivido 11 abortos. Ella indica que solo pudo tener un hijo, que nació antes de haber ingresado como trabajadora bananera. Seguidamente, se reproduce este relato.

Relato 5. Documento sin fecha de nacimiento

Trabaje en la vananera y fuí afectada por el nemagón y este me cauzo mi esterilidad yo antes de aber trabajado con la vananera abía tenido un Hijo luego que trabaje en la vananera solo quedava embarazada y abortaba de allí en adelante nunca pude tener o pegar un embarazo. 
llegaba a 4 y asta 6 meces y luego me agarraban picazones vaginales ronchas $y$ dolores de estomago agudos $y$ me agarraba descompociciones $y$ frio el cuerpo $y$ comenzaba a botar agua $y$ sangrados $y$ acudia al medico y susedia lo de ciempre perdida de embarazo me asian legrado $y$ fin $y$ asi susesivamente $y$ nunca pude tener otro Hijo, tuve 11 abortos o embarazos. 8 legrados.

Padesco dolores de vientre agudo picasones dolorozas, descomposiciones, corta de vista desde entonces. aveces me pongo en una tembladera cuando me viene la regla (sERPaJ, 1996).

En el relato 40 , la mujer que escribe señala haber tenido 5 abortos, una hija muerta al nacer y 2 hijas vivas. Al igual que en los relatos anteriores, se indican otros padecimientos desarrollados en el cuerpo de esta mujer.

Relato 40. Documento sin fecha de nacimiento

Empeza a trabajar en el 79 hasra el 85-Standart Fruit Company.

en la planta $=$ deflorar. Sello... todo tipo y en el campo a quitar hierba...

Nemagon $y$ otros

- dolor de huesos (manos y pies inchados...)

- dolores de garganta (dicen los doctores que es una alergia permanente).

- dolores de cabeza permanentes.

He tenido 5 abortos (no mayor de 3 meses) y 1 niña murió 8 horas despues de nacer

2 hijas

[Nombre de la hija] (24) = dolores de cabeza, garganta, huesos.

La menor de 21 anos tiene menos problemas.

Mi hermano era embolsador de la Standart y murió de cáncer en la garganta.

Tengo otro hermano (apuntado en el Comité) es estéril (SERPAJ, 1996).
En algunos casos, la historia de los abortos se establece con más claridad en la memoria que se construye $y$ se presenta en las palabras recuperadas en los documentos, como se puede observar en el relato 15 . En este escrito, la mujer va narrando el historial de sus partos, en donde tenía hijos vivos o abortos, lo que también se encuentra presente en el relato 5, reproducido anteriormente.

Relato 15. Mujer nacida el 28 de octubre de 1956.

Llo [...nombre de la mujer...] edad 43 años en el año 69 travaje en santa clara en aquel tiempo se llamava fica vieja entre sellando despues empacando y fulmigando la fulmigasion se asia para volsas de rasimos de banano asta el 71 fue el año que salí asta entonces mí primer ijo lo tuve en el 72 afectado con asma segundo una niña muerta tersero un avorto cuarto una niña viva pero con asma quinto otra niña con aficsia pero el doctor dijo asma y quinto niño con asma los padesimientos míos son mas el asma lla que cuando estaba envarasada me afectava mas $y$ otros males pero no tomava importancia (SERPAJ, 1996).

En un último relato, que se presenta seguidamente, la mujer señala haber tenido dos pérdidas.

Relato 12. Documento sin fecha de nacimiento

Trabaje en finca Carolina le ayudava a enjugar la ropa y abriendo bolsa $y$ enllenando manos.

Padecimientos dolor de cabeza de la sangre dolor de riñones preción alta mareos niños vivos $7 y$ de los 7 tengo una niña embalidad $y$ despues de ella tuve 1 avorto $y$ ace como 4 años tuve otro pero ese se me murio en el vientre a los 7 meses de envaraso lo tuve en el espital del carrderón (sERPAJ, 1996).

Estos documentos son sumamente importantes para el proceso de reconstrucción de la memoria de estas mujeres, a través del registro 
de sus abortos. Los documentos no permiten dar cuenta de las formas en las que estas mujeres elaboraron sus pérdidas, dado que no era la finalidad de los encuentros, pero abren la posibilidad de posicionar el dolor y el sufrimiento al que se vieron enfrentadas, en un espacio colectivo de intercambios. Es muy probable que estas mujeres vivieran estas experiencias solas, sin ningún tipo de acompañamiento $y$ mucho menos, sin tener la posibilidad y la apertura de las organizaciones sindicales para plantear estas situaciones en forma de reivindicaciones políticas frente a las compañías bananeras. Lamentablemente, a partir de los documentos estudiados, no es posible conocer si estas mujeres se encontraban vinculadas a este tipo de organizaciones, dado que esta no se consignó.

Ahora bien, en la entrevista realizada, la mujer entrevistada señaló que ella no se vinculó a organizaciones de este tipo, dado que, "los que más peleaban eran los hombres" (Comunicación personal, extrabajadora bananera, 20/07/2016). En esta entrevista, la mujer indicó que muchos de estos abortos no eran atendidos en los hospitales públicos, debido a que si las mujeres no se encontraban muy afectadas por la pérdida sufrida, ellas no asistían a los hospitales o eran atendidas en sus casas por "señoras" que se encargaban de estas labores. Adicionalmente, tal y como se menciona en la literatura consultada, no todas las mujeres tenían acceso al seguro social, como se observa en la siguiente transcripción:

Seguro iban al seguro, si se veían mal, porque si no, no, pasaba, porque yo fui una, yo tuve un aborto como a los, de trabajar en la finca, como a los cinco o seis años. Diay, como era pequeñito, no estaba, la señora que me atendió en la casa, ya está, en la casa, te curaste, te pusieron pastillas que daban antes las señoras, porque yo, en el hospital el único que fui a tener es el varón, el papá de ese chiquito $\left[. .{ }^{13} . ..\right]$, porque yo en ese tiempo, antes no tenía seguro, ni tampoco trabajaba en

13 Hace referencia a un nieto que está cerca en el momento en el que se realiza la entrevista. las bananeras, ni habían aquí, las bananeras vinieron aquí en el sesenta y nueve y el último que tuve yo, que fue el varón fue en el setenta y cuatro, ya trabajaba en las bananeras que fue el que apareció enfermo. Y ese sí, como tenía seguro e incapacidad entonces sí fui al seguro. Todos fueron en la casa. Esa pérdida que tuve, que comenzó, diay seguro no estoy embarazada y comenzó a venirme la regla, a venirme la regla y venirme, cuando fueron a traer una señora de esas para que le adivinaran a uno. Y dice, "pero ya, ya, ya está buena, ya lo botó (Comunicación personal, extrabajadora bananera, 20/07/2016).

A partir de esta reproducción, se puede plantear la hipótesis de que muchos de los abortos que se pudieron haber presentado en la zona, vinculados a la exposición a este agroquímico, no quedaron registrados en los datos recopilados por los centros de salud, debido a lo frecuente que pudo haber sido la atención de estas pérdidas en las casas de las trabajadoras bananeras. Sin embargo, esta es una explicación tentativa que necesita de mayor investigación.

\section{EL NEMAGÓN COMO EXPLICACIÓN DE TODOS LOS PADECIMIENTOS}

Ahora bien, es importante señalar que hay una serie de relatos que apuntan a padecimientos que no necesariamente se encuentran relacionados con la afectación por el nemagón, pero que, en el marco de las explicaciones que las mujeres brindan, este aparece como el elemento que permite entender el origen de dichos padecimientos. El relato 1 ejemplifica lo argumentado.

Relato 1. Documento sin fecha de nacimiento

travaje en la finca el Prado en el año 1971 por espacio de 1 año tambien travaje en la finca estación experimental los Diamantes

Mis enfermedades son dolor de cabeza mareo falta de visión dolor de todos los huesos 
Soi operada de una mano por los mismos dolores

Soi operada de la visicula

Tengo un riñon duro y el otro con un criste se me izo una gastritis crónica

Me sale de los ojos una cosa gomosa pero cristalina $y$ me arde

En esta finca travaje 9 años $1 / 2$

cuando travaje en los Diamantes estuve en barasada y mi hijo nacio con problemas es sordo y mudo y no soporta los olores a químicos el nacio en 1974 se llama [...nombre del hijo...] Tengo una hija de 29 años no travajo pero me iva dejar el almuerzo al travajo ella era muy saludable ace unos años atrás se a estado poniendo muy flaquita y sus hijas padecen mucho de asma (sERPAJ, 1996).

Otro relato que puede ser leído a partir de esta línea de interpretación es el que se presenta seguidamente.

Relato 27. Documento sin fecha de nacimiento

Trabaje en finca carolina en el 69 o 70 al 80. Yo trabajaba en la planta de selector, demanaba, fumigaba.

Tengo 2 hijos, uno de 8 años me padece de tiroide y asma y la otra de 6 años, nacio prematura con mucho problema de nutrición, crecimiento, e tenido 2 abortos y Actualmente no puedo tener un bebe saludable padesco de asma, fui operada de glándulas de eso mismo, reumatismo, mis hijos tambien padecen de problemas en la piel, padesco de la vista, mareos (SERPAJ, 1996).

En ambos casos, los padecimientos son explicados por la exposición al agroquímico, a pesar de que las problemáticas de salud trascienden los efectos que dicha sustancia pudiera tener. Si bien, puede existir un vínculo directo entre una lógica productiva que produce desigualdad y efectos en los cuerpos, esto no significa que el nemagón fuese el causante de problemas como gastristis o desnutrición, los que probablemente tienen una relación directa con problemas de acceso a una alimentación saludable, más que con el agroquímico. Lo mismo se puede decir de los relatos en los que las mujeres señalan tener un desgaste en la columna, lo que pudo ser ocasionado por las labores características de su puesto de trabajo y no necesariamente por la exposición al nemagón.

En este sentido, el nemagón se ha convertido en una especie de explicación, socialmente legitimada $y$ justificada en el marco de las movilizaciones realizadas por este tipo de actores, para exponer denuncias en torno a las expresiones de la desigualdad en el cuerpo, que no necesariamente tienen vínculo con las consecuencias concretas de un determinado agroquímico; aunque en términos generales, efectivamente, pueden tener relación con la lógica de los monocultivos y las consecuencias sociales. Lo argumentado no niega la importancia que tiene este registro en la reconstrucción social del dolor y del sufrimiento ambiental (Auyero y Swistun, 2008; Kleinman y Kleinman,1997), justamente, porque las explicaciones construidas trascienden los efectos del agroquímico.

\section{ALGUNAS IDEAS PARA CONCLUIR}

Las investigaciones sobre la exposición a los agroquímicos muestran cómo a pesar de que el riego de los agroquímicos ha sido un trabajo tradicionalmente asociado a los hombres, las mujeres han realizado este tipo de labores y se han encontrado expuestas a estas sustancias, lo cual las ha llevado a desarrollar una serie de malestares físicos y enfermedades. En este contexto, los problemas enfrentados por las mujeres se relacionan con los altos niveles de analfabetismo que dificultan leer la información de las sustancias que utilizan, etiquetas e instrucciones que se encuentran en un idioma distinto al suyo, además de acceso limitado a equipos o implementos de seguridad. Cuando las mujeres se ven afectadas de manera directa, en muchas ocasiones no atienden su salud dado que, en caso de que la intoxicación sea leve, no reconocen los síntomas o los confunden con otras enfermedades; al no tener acceso a seguro 
social o centros médicos cercanos, atienden sus malestares con remedios caseros.

La experiencia que se recupera en este artículo da cuenta de algunos puntos de encuentro en relación con lo planteado en la literatura consultada. La experiencia propiciada por SERPA posibilitó un registro de la afectación de las mujeres vinculadas a la actividad bananera, en donde distintos malestares físicos, entre los que destacan los abortos, fueron señalados como las principales consecuencias de su trabajo. Este registro resulta de suma importancia a fin de posicionar el rol de las mujeres en la producción bananera, con las consecuencias que este trabajo tuvo en sus cuerpos y en los cuerpos de sus hijas e hijos. Sin embargo, es necesario llevar a cabo un estudio a profundidad en torno las consecuencias de estas enfermedades y pérdidas en su salud mental, así como, las estrategias subjetivas $y$ sociales para hacerle frente al sufrimiento ambiental, aspectos no abordados en este texto.

Uno de los puntos de encuentro entre los estudios de caso y la experiencia estudiada se relaciona con el acceso a los servicios de salud de las mujeres dedicadas a las labores agrícolas, así como, la atención de las enfermedades en estos espacios. Es probable que las mujeres guardaran silencio en relación con sus padecimientos $y$ enfermedades, $y$ los atendidos en hospitales públicos fueron casos extremos. En la atención de los abortos, por ejemplo, "las señoras" dedicadas a estas labores, probablemente parteras, jugaron una labor importante en este proceso, por lo que el subregistro de este tipo de expresiones puede ser muy alto.

Ahora bien, lo que estaba ocurriendo en los cuerpos de las mujeres pudo haber sido un elemento aglutinador sumamente valioso para la organización y articulación de demandas a nivel de organizaciones sindicales, sin embargo, esto no fue lo que sucedió. A manera de hipótesis se puede plantear que esta problemática surgió, no solo en un contexto de debilitamiento del sindicalismo bananero (Mora, 2016b), si no en el marco de una organización política controlada por hombres, profundamente machista (AsEPRoLA, 2003; Frank, 2006), incapaz de registrar el dolor y el sufrimiento de estas mujeres. De esta forma, el silencio, el debilitamiento sindical y el machismo organizativo pudieron ser parte de una mezcla que impidió que el sufrimiento de las mujeres se convirtiera en una demanda fuerte dentro de las organizaciones sindicales.

En este contexto, en los encuentros propiciados por SERPAJ, las mujeres encontraron y construyeron un espacio para darle lugar al dolor compartido con otras mujeres, aunque no necesariamente fuera un dolor hecho público. Esta explicación vendría a reafirmar la idea de que en el movimiento de trabajadores bananeros afectados por el nemagón, las demandas masculinas se impusieron en la jerarquía de reivindicaciones, desconociendo el sufrimiento de sus compañeras trabajadoras.

\section{REFERENCIAS}

Asociación de Servicios de Promoción Laboral (aseprola). (2003). Lo que hemos vivido: luchas de mujeres bananeras. San José: Asociación de Servicios de Promoción Laboral (ASEPROLA).

Auyero, J. (2004). Vidas Beligerantes. Dos mujeres argentinas, dos protestas y la búsqueda de reconocimiento. Argentina: Editorial de la Universidad Nacional de Quilmes.

Auyero, J. y Swistun, D. (2008). Inflamable. Estudio del sufrimiento ambiental. Argentina: Paidós Editorial.

Barcellos, G.; Batista, S. (2007). Mulheres e eucalipto. Histórias de vida e resistência. Impacto da monocultura de eucalipto sobre mulheres indígenas e quilombolas no Espírito Santo. Uruguay: Movimento Mundial pelas Florestas Tropicais.

Camarena, L.; von Glascoe, C.; Martínez, C.; y Arellano, E. (mayo-agosto, 2013). Riesgos del trabajo y salud: percepción de mujeres jornaleras en el noreste de México. Salud Colectiva (9) 2, 247-256.

Cárdenas, R. (s.f.). Informe de Organización y perspectivas de los casos y consecuencias en las personas afectadas por el Nematicida aplicado por las compañías bananeras 
en Costa Rica. Pasantía de la Escuela de Sociología de la Universidad Nacional (una)-Servicio Paz y Justicia (serPas).

Das, V. (2008). Lenguaje y cuerpo: transacciones en la construcción del dolor. En Ortega, F. (ed.) Veena Das: sujetos de dolor, agentes de dignidad. Bogotá: Universidad Nacional de Colombia; Pontificia Universidad Javeriana; Instituto Pensar.

Dinham, B. (2003). Introduction. En Jacobs, M. \& Dinham, B. (ed.). Silent Invaders, pesticides, livelihoods and women's health. Londres: Zed books.

Frank, D. (2006). El poder de las mujeres es poder sindical. La transformación de los sindicatos bananeros en América Latina. Honduras: Editorial Guaymuras.

Giralt, M. (1996). La problemática ética del uso del рвср en Costa Rica. Revista de Filosofía xxxiv (83-84), 415-421.

González, E. (2014). Las mujeres en la industria colombiana de las flores. Observatorio de Multinacionales en América Latina (омац). Informe Omal $\mathrm{N}^{\circ} 11$.

Jacobs, M. \& Dinham, B. (2003). Silent Invaders, pesticides, livelihoods and women's health. Londres: Zed Books.

Kleinman, A. y Kleinman, J. (1997). The Appeal of Experiences; The dismay of Images: Cultural Appropiations of Suffering in Our Times. Social Suffering. California: University of California Press.

Korovkin, T. (2004). Globalización y pobreza. Los efectos sociales del desarrollo de la floricultura de exportación. En Korovkin, T. (comp.). Efectos sociales de la globalización. Petróleo, bananos $y$ flores en Ecuador. Ecuador: Centro de Investigación de los Movimientos Sociales del Ecuador (cedime).

Mora, S. (2013). Reflexiones para el análisis comparativo de movimientos sociales: El caso de extrabajadoras y extrabajadores bananeros afectados por el nemagón en Costa Rica y Nicaragua. Anuario de Estudios Centroamericanos, 39, 211-232.

Mora, S. (2016a). Entre la movilización y la desmovilización política: Reflexiones metodológicas sobre desigualdad, sufrimiento y movimientos sociales. Revista de Ciencias Sociales. Artículo en proceso de evaluación.

Mora, S. (2016b). Las consecuencias del "oro verde". Sufrimiento ambiental y el movimiento de las trabajadoras y los trabajadores bananeros afectados por el nemagón. Documento en proceso de evaluación.

Nivia, E. (2000). Mujeres y plaguicidas. Una mirada a la situación actual, tendencias y riesgos de los plaguicidas. Estudio de caso en Palmira. Colombia. Colombia: Rapalmira.

Reeves, M.; Murphy, T. \& Calvo, T. (2003). Farmworker Women and Pesticides in California's Central Valley. San Francisco: Pesticides Action Network North America (PanNa).

Servicio Paz y Justicia (serpaj) (1996). Archivo. Costa Rica.

Siliprandi, E. (2011). Mulheres Agricultoras no Brasil: sujeitos políticos na luta por soberania e segurança alimentar. Pensamiento Iberoamericano 9, 169-184.

Valdés, X. (2015). Feminización del empleo y trabajo precario en las agriculturas latinoamericanas globalizadas. Cuadernos de Antropología Social, (41), 39-54.

Van Wendel, B.; Mora, A.; Córdoba, L.; Cano, J.; Faniband, M., Wesseling, C.; Ruepert, C.; Öberg, M.; Quesada, R.; Brenda, E. y Lindh, C. (2014). Aerial Application of Mancozeb and Urinary Ethylene Thiourea (етu) Concentrations among Pregnant Women in Costa Rica: The Infants' Environmental Health Study (IsA). Environmental Health Perspectives, 122 (12), 1321-1329.

Zuluaga, G. y Arango, C. (2013). Mujeres campesinas: resistencia, organización $y$ agroecología en medio del conflicto armado. Cuadernos de Desarrollo Rural, 10 (72), 159-180.

Fecha de ingreso: 12/12/2016 Fechas de aprobación: 15/03/2017 